\section{Ist eine platinhaltige Kombination bei diesen Patienten sicher?}

Minegishi $Y$ et al. The safety and efficacy of weekly paclitaxel in combination with carboplatin for advanced non-small cell lung cancer with idiopathic interstitial pneumonias. Lung Cancer 2011;71:70-74

\section{Hintergrund}

Bei Patienten mit einer idiopathischen Lungenfibrose (IPF) ist die Inzidenz eines Bronchialkarzinoms mit einem relativen Stellenwert einer palliativen Chemotherapie bei Patienten mit Bronchialkarziinterstitieller Pneumonie (IIP) unklar.

\section{Methodik}

In eine japanische prospektive Studie wurden 18 Patienten mit lokal fortgeschrittenem, inoperablem $(n=5)$ bzw. metastasiertem, nicht kleinzelligem $(\mathrm{n}=13)$ Bronchialkarzinom und fibrosierender Lungenerkrankung (14 Männer, 4 Frauen; Durchschnittsalter: 71 Jahre; 33-81 Jahre) aufgenommen. Histologisch lagen Plattenepithelkarzinome $(n=7)$, Adenokarzinome $(\mathrm{n}=6)$ und undifferenzierte nicht kleinzellige $(\mathrm{n}=5)$ Bronchialkarzinome vor. $83 \%$ der Patienten waren (Ex-)Raucher. Sechs Patienten hatten eine histologisch gesicherte idiopathische Lungenfibrose (IPF) und zwölf Patienten einen fibrosierenden Lungengerüstprozess anderer Ätiologie („Non-IPF“). Die Vitalkapazität lag durchschnittlich bei $82 \%$ des eine Langzeitsauerstofftherapie.

Die Behandlung erfolgte mit Carboplatin (AUC 5,0; vierwöchentlich und Paclitaxel (100 mg/m² ; wöchentlich) über drei Wochen.

Der primäre Endpunkt der Studie war das Auftreten einer akuten Exazerbation (AE) der fibrosierenden Lungenerkrankung als chemotherapieinduzierte Therapiekomplikation. Eine AE wurde definiert als eine Zunahme der Dyspnoe innerhalb von vier Wochen mit radiologischem Nachweis von neu aufgetretenen Milchglasinfiltraten und einem Abfall des Sauerstoffpartialdrucks $\left(\mathrm{paO}_{2}\right)>10 \mathrm{mmHg}$ Risiko von 7-14 erhöht. Derzeit ist der nom und gleichzeitiger idiopathischer Sollwerts. Keiner der Patienten erhielt bei Ausschluss kardialer oder infektiöser Ursachen. Sekundäre Endpunkte waren die Toxizität, das Therapieansprechen, das mediane progressionsfreie Überleben (PFS) und das Gesamtüberleben.

\section{Ergebnisse}

Die Gesamtansprechrate (komplettes oder partielles Ansprechen) betrug 61\%.

Eine therapieinduzierte tödliche AE wurde in einem Fall beobachtet $(5,6 \%$; 95\%-Konfidenzintervall: 0-17\%) und trat erst sieben Wochen nach Abschluss des vierten Chemotherapiezyklus auf. Im Verlauf einer Zweitlinientherapie erlitten drei Patienten (Docetaxel: $\mathrm{n}=2$; Gefitinib: $\mathrm{n}=1$ ) eine tödliche $\mathrm{AE}$. Die häufigste Hämatotoxität war eine Neutropenie Grad 3/4 (33\%). Eine fieberhafte Neutropenie trat bei einem Patienten auf.

Das mediane PFS betrug 5,3 Monate und die 1-Jahres-Überlebensrate $22 \%$.

\begin{tabular}{l|l}
\hline $\begin{array}{l}\text { Akute Exazerbationen } \\
\text { und Responseraten }\end{array}$ & Tabelle 1 \\
\hline $\begin{array}{l}\text { Patienten } \\
\text { Pa }\end{array}$ & 18
\end{tabular}

\section{Patienten}

Akute Exazerbationen

Therapiebedingt

- davon tödlich

Bedingt durch die Second-line-Therapie

\pm - davon tödlich

Unabhängig von der Therapie

- davon tödlich

3

1

Objektives Ansprechen

Komplettes und partielles Ansprechen

Stabilisierung

Fortschreiten der Erkrankung

Nicht auswertbar

Gesamtansprechrate (\%)

\section{Kommentar}

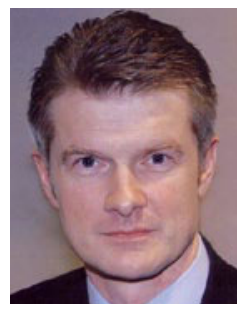

Prof. Dr. med.

Martin Kohlhäufl,

Stuttgart-Gerlingen
Eine IIP ist ein unabhängiger Risikofaktor (relatives Risiko 4,8-5,6) für eine akute Exazerbation der Grunderkrankung bei der Durchführung einer antineoplastischen Chemotherapie unabhängig von der Wirkstoffgruppe [3]. Die Rate einer gefitinibinduzierten Lungentoxizität per se wird bei japanischen Patienten mit 3,98\% angegeben und liegt damit 13-fach höher als in den USA $(0,3 \%)$.

In dieser ersten prospektiven Pilotstudie erlitt ein Patient $(5,6 \%)$ mit einer histologisch gesicherten idiopathischen Lungenfibrose eine akute tödlich verlaufende therapieassoziierte Exazerbation (AE) der fibrosierenden Lungenerkrankung. Zum Vergleich liegt die Rate einer AE bei unbehandelten IIP per se bei $8,5-14 \%[4,5]$.

Somit bestätigt diese Pilotstudie retrospektive Untersuchungen, die zeigten, dass die Kombinationstherapie mit Carboplatin und Paclitaxel bei NSCLC und IIP eine therapeutische Option darstellen könnte. Dies muss jedoch noch an größeren Patientenkollektiven validiert werden.

\section{Literatur}

1. NCCN Clinical Practice Guidelines in Oncology V.2.2009 (www.ncen.org)

2. Baumgarner KB et al. Cigarette smoking. A risk factor for idiopathic pulmonary fibrosis. Am J Resp Crit Care Med. 1997;155:242-248

3. Kudoh $\mathrm{S}$ et al. Interstitial lung disease in Japanese patients with lung cancer: a cohort and nested case-control study Am J Respir Crit Care Med. 2008;177:1348-1357

4. Kim DR et al. Acute exacerbation of idiopathic pulmonary fibrosis: frequency and clinical features. Eur Respir J. 2006;27:143-150

5. Azuma A et al. Double-blind, placebo-controlled trial of pirfenidone in patients with idiopathic pulmonary fibrosis. Am J Respir Crit Care Med. 2005;171:1040-1047

6. Minegishi $Y$ et al. Exacerbation of idiopathic interstitial pneumonias associated with lung cancer therapy. Intern Med. 2009;48:665-672 